\title{
Risikoabschätzung für Fehlbildungen in der Schwangerschaft: Dosisabhängigkeit beachten!
}

Auswertungen von Schwangerschaftsregistern ermöglichen eine vergleichende Bewertung des Fehlbildungsrisikos verschiedener Antiepileptika bei Einnahme in der Schwangerschaft. Parallel existieren mehrere solcher Register, zum Beispiel das europäische (EURAP) und das nordamerikanische (NAAED). Hernández-Diaz et al. haben Daten aus dem nordamerikanischen Register von Frauen mit einer antiepileptischen Monotherapie im ersten Trimenon analysiert - und nur für Valproat eine Dosisabhängigkeit festgestellt.

$\mathrm{n}$ das nordamerikanische Schwangerschaftsregister NAAED werden Frauen mit Epilepsie unter antiepileptischer Therapie zu Beginn beziehungsweise während der Schwangerschaft eingeschlossen; etwa drei Monate nach Entbindung wird die Häufigkeit großer Fehlbildungen der Kinder der Studiengruppe dokumentiert. Durch einen fortlaufenden Studienein-

Tabelle 1

\begin{tabular}{|c|c|c|}
\hline & $\begin{array}{l}\text { Absolutes } \\
\text { Risiko }\end{array}$ & $\begin{array}{l}\text { Relatives } \\
\text { Risiko }\end{array}$ \\
\hline Valproat & $\begin{array}{l}9,3 \% \\
\text { (30 von 323) }\end{array}$ & 5,1 \\
\hline Phenobarbital & $\begin{array}{l}5,5 \% \\
\text { (11 von 199) }\end{array}$ & 2,9 \\
\hline Topiramat & $\begin{array}{l}4,2 \% \\
\text { (15 von 359) }\end{array}$ & 2,2 \\
\hline $\begin{array}{l}\text { Carbam- } \\
\text { azepin }\end{array}$ & $\begin{array}{l}3,0 \% \\
\text { (31 von 1033) }\end{array}$ & 1,5 \\
\hline Phenytoin & $\begin{array}{l}2,9 \% \\
(12 \text { von } 416)\end{array}$ & 1,5 \\
\hline Levetiracetam & $\begin{array}{l}2,4 \% \\
\text { (11 von } 450)\end{array}$ & 1,2 \\
\hline Lamotrigin & $\begin{array}{l}2,0 \% \\
\text { (31 von 1562) }\end{array}$ & Referenz \\
\hline $\begin{array}{l}\text { Gesunde } \\
\text { Kontrollen }\end{array}$ & $\begin{array}{l}1,1 \% \\
\text { (5 von 442) }\end{array}$ & \\
\hline
\end{tabular}

schluss nimmt die Datenbasis, insbesondere auch hinsichtlich der neueren Antiepileptika, ständig zu, so dass durch regelmäßige Auswertungen zunehmend robustere Risikoabschätzungen erfolgen können.
Hernández-Díaz et al. haben Daten von 4.899 Patientinnen untersucht, die von 1997 bis 2011 in das NAAED aufgenommenen wurden. Alle Patientinnen wurden im ersten Schwangerschaftstrimester mit einer antiepileptischen Monotherapie behandelt. Die Forscher ermittelten das absolute Risiko für große Fehlbildungen einzelner Antiepileptika und verglichen dies mit einer registerinternen Kontrollgruppe von 442 Frauen ohne Epilepsie und Antiepileptika. Zusätzlich präsentierten die Autoren die relativen Risiken einzelner Antiepileptika verglichen mit Lamotrigin-Monotherapie. Dieser Vergleich soll die klinisch relevante Frage beantworten, welches Antiepileptikum das sicherste ist. Eine aktive (Lamotrigin-) Vergleichsgruppe hat den Vorteil, dass dadurch die meisten konfundierenden Einflüsse kontrolliert werden können. Die ermittelten absoluten und relativen Risiken sind in Tabelle 1 dargestellt, eine Dosisabhängigkeit wurde nur für Valproat gefunden.

Kommentar: Diese Studie ist für die Beratung von Epilepsiepatientinnen mit Kinderwunsch sehr wichtig und liefert aktuelle Daten für einen Vergleich der Sicherheit mehrerer, einschließlich einiger neuer, Antiepileptika. Leider gehen die Autoren auf eine ebenfalls kürzlich erschienene Auswertung des europäischen Schwangerschaftsregisters EURAP nicht ein (Tomson et al. [1]). Auch wenn die Ergebnisse beider Register aufgrund unterschiedlicher Methodiken nur schwer vergleichbar sind, scheint sich zumindest die Rangfolge der Risiken, von Valproat und Phenobarbital über Carbamazepin bis hin zu Lamotrigin zu entsprechen. Tomson et al. [1] betonen aber den wichtigen Punkt der Dosisabhängigkeit: Während sich in der nordamerikanischen Studie nur für Valproat dosisabhängige Effekte zeigten, fanden sich diese in der EURAP-
Studie für jedes der untersuchten Medikamente (Carbamazepin, Lamotrigin, Valproat, Phenobarbital). Eine wichtige Aussage der EURAP-Studie ist daher, dass Risikoabschätzungen ohne Berücksichtigung der Dosis kaum möglich sind: So ist beispielsweise Carbamazepin in sehr hoher Dosis ( $\geq 1.000$ $\mathrm{mg}$ ) mit einem höheren Risiko für Malformationen verbunden als Valproat in niedriger Dosis (<700 mg, [1]).

Der Komplexität der Zusammenhänge und auch der Registerauswertungen, in denen sehr viele Einflussgrößen parallel kontrolliert werden müssen, kann hier aus Platzmangel nicht annähernd entsprochen werden. Zudem ist zu berücksichtigen, dass Antiepileptika (insbesondere Valproat, siehe [2]), während der Schwangerschaft auch Risiken für die Intelligenzentwicklung des Kindes mit sich bringen können. Trotz der hohen Komplexität der Thematik ist die differenzierte Kenntnis der Datenlage für die Beratung von schwangeren Patientinnen oder von Patientinnen mit Kinderwunsch unabdingbar. An den regelhaften Registereinschluss von Frauen, die zum Zeitpunkt der Konzeption Antiepileptika einnehmen, sollte gedacht werden (www.eurap. de).

Dr. rer. nat. Martin Lutz

\footnotetext{
Referenzen:

1. Tomson T et al. EURAP study group. Dosedependent risk of malformations with antiepileptic drugs: an analysis of data from the EURAP epilepsy and pregnancy registry. Lancet Neurol. 2011 Jul; 10 (7): 609-17

2. Meador KJ et al. NEAD Study Group. Effects of fetal antiepileptic drug exposure: outcomes at age 4.5 years. Neurology. 2012 Apr 17; 78 (16): 1207-14
}

Hernández-Díaz S et al. For the North American AED Pregnancy Registry; North American AED (Antiepileptic Drug) Pregnancy Registry; Scientific Advisory Committee; North American AED Pregnancy Registry. Comparative safety of antiepileptic drugs during pregnancy. Neurology. 2012 May 22; 78 (21): 1692-99 